pp 91-145, Academic Press, New York.

Tsuboi, M., Nishimura, Y., Hirakawa, A. Y., \& Peticolas, W. L. (1987) in Biological Applications of Raman Spectroscopy (Spiro, T. G., Ed.) John Wiley \& Sons, New York.

Tucker, J., Sczakiel, G., Feurstein, J., John, J., Goody, R. S., \& Wittinghofer, A. (1986) EMBO J. 5, 1351-1358.

Wittinghofer, A., Warren, W. F., \& Leberman, R. (1977) FEBS Lett. 75, 241-243.
Wooley, P., \& Clark, B. F. C. (1989) Bio/Technology 7, 913-920.

Yue, K. T., Yang, J. P., Charlotte, M., Lee, S. L., Sloan, D., \& Callender, R. (1984) Biochemistry 23, 6480-6483.

Yue, K. T., Deng, H., \& Callender, R. (1989) J. Raman Spectrosc. 20, 541-546.

Yue, K. T., Yang, J. P., Charlotte, M., Lee, S. K., Sloan, D., \& Callender, R. (1984) Biochemistry 23, 6480-6483.

\title{
RNA Folding during Transcription by T7 RNA Polymerase Analyzed Using the Self-Cleaving Transcript Assay ${ }^{\dagger}$
}

\author{
Kamala Tyagarajan, Joseph A. Monforte, and John E. Hearst* \\ Department of Chemistry, University of California, Berkeley, and Laboratory of Chemical Biodynamics, Lawrence Berkeley \\ Laboratories, Berkeley, California 94720 \\ Received July 3, 1991; Revised Manuscript Received August 27, 1991
}

\begin{abstract}
We have used a self-cleaving RNA molecule (a "hammerhead") to study the length-dependent folding of RNA produced during transcription by T7 RNA polymerase. Transcript elongation is arrested at defined positions using chain-terminating ribonucleoside triphosphate analogues, 3 '-deoxynucleoside triphosphates. When the nascent transcript attains the minimum length required for the "hammerhead" domain of the transcript to fully emerge from the ternary complex, the "hammerhead" structure forms and self-cleaves, producing a truncated product. The experiment yields an RNA sequencing ladder which terminates at the length at which cleavage becomes possible; the sequencing ladder is compared to that generated by using a noncleaving control template. We have shown that 13 nucleotides past the cleavage point must be synthesized before the transcript can self-cleave in the ternary complex whereas RNA freed from the complex by heating can cleave with only 3 or more nucleotides present beyond the cleavage site. The results indicate that the RNA in T7 RNA polymerase is not free of steric interactions in the ternary complex and not available for structure formation until it is at least 10 bases away from the site of polymerization. The results suggest that the maximum possible length of the RNA-DNA hybrid in the ternary complexes is 10 . The relevance of the results in comparisons with other RNA polymerases, especially Escherichia coli RNA polymerase, is discussed.
\end{abstract}

$\mathbf{T}_{7}$ RNA polymerase is a small, monomeric bacteriophage enzyme with a molecular weight of about 100000 which is highly specific for the transcription of its well-conserved promoters (Chamberlin \& Ryan, 1982). T7 RNA polymerase shares homology with T3, SP6, and yeast mitochondrial RNA polymerases (Masters et al., 1987). In contrast to the larger, multiple-subunit bacterial and eukaryotic RNA polymerases, T7 RNA polymerase is much less complex both structurally and functionally and carries out all the steps of transcription by itself in vitro without the aid of additional protein factors (Coleman, 1974). These components combine to make $T 7$ RNA polymerase an ideal system in which to study the "minimum apparatus" for transcription.

Our studies concern the structure and dynamics of the T7 RNA polymerase.RNA.DNA ternary complex during the elongation phase of transcription. Our experiments address the question of when the nascent RNA transcript becomes free of steric interactions in the ternary elongation complex and

\footnotetext{
${ }^{\dagger}$ This work was supported by NIH Grant GM41911 to J.E.H. and by Contract DE-AC03-76SF00098 from the Director, Office of Energy Research, Office of General Life Sciences, Structural Biology Division of the U.S. Department of Energy.

* To whom correspondence should be addressed at the Department of Chemistry, Hearst Research Group, University of California, Berkeley.
}

is available to form structure with itself. This focus allows us to estimate the maximum possible length of the RNADNA hybrid in the ternary elongation complex. It also provides information about the importance of RNA structure formation in processes of pausing and termination which require RNA structure to form within a few nucleotides of the site of polymerization (von Hippel et al., 1984; Yager \& von Hippel, 1991).

Footprinting studies have established that between 13 and 19 nucleotides of the DNA are protected by T7 RNA polymerase during elongation (Shi et al., 1988). This establishes an upper limit to the size of the RNA-DNA hybrid. The size of the unwound region of the DNA in the Escherichia coli RNA polymerase ternary complex is 17 nucleotides, and the length of the RNA-DNA hybrid is 12 nucleotides (Gamper \& Hearst, 1982; Hanna \& Meares, 1983; Monforte et al., 1990). Because T7 RNA polymerase is a smaller and simpler enzyme than $E$. coli RNA polymerase, one would expect that the maximum size of the RNA-DNA hybrid in the ternary complex would be either the same or smaller.

Several groups have examined the contacts and accessibility of the nascent RNA chain in order to determine the length of the RNA-DNA hybrid. Hanna and Meares (1983) have used cleavable photoaffinity labeling techniques to show that 
A. DNA Template
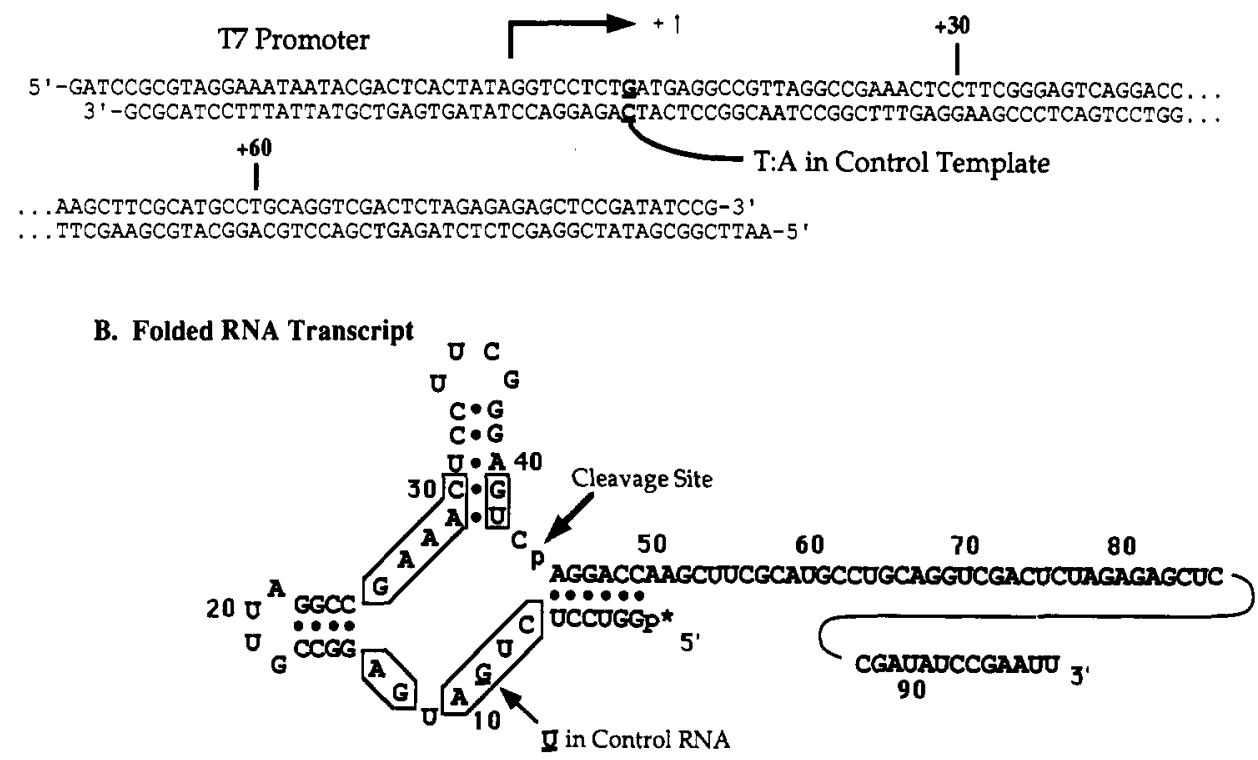

FIGURE 1: Templates and transcript sequences. (A) Sequence of the synthetic double-stranded template, containing a modified T7 class III promoter followed by a sequence coding for the "hammerhead" structure. The "hammerhead" region is followed by a region of minimum complementarity to the "hammerhead" sequence. The control template contains a base pair change at +9 coding for a $U$ instead of a $G$ in the resulting RNA product. This mutation destroys autolytic activity within the RNA. (B) The full-length RNA product: The "hammerhead", shown in its proposed secondary structure, cleaves autolytically between nucleotides $C_{43}$ and $A_{44}$ when the "hammerhead" structure is fully formed. The conserved bases required for activity, as determined phylogenetically, are enclosed in boxes. Most deviations from this consensus sequence result in the loss of activity, as is the case for the control transcript. In addition to the conserved nucleotides, the three helical stems shown must form to promote self-cleavage. Constraints on the numbers and types of base pairs in these three stems are minimal though different sequences do exhibit a wide range of cleavage rates (Fedor \& Uhlenbeck, 1990).

the $5^{\prime}$ end of a growing RNA chain, within the $E$. coli RNA polymerase ternary complex, labels the DNA coding strand for 12-14 base pairs upstream from the point of synthesis. RNase protection experiments by Kumar and Krakow (1975) on the transcriptional complex of Azotobacter vinelandii, which is closely related to $E$. coli $\mathrm{RNA}$ polymerase, estimated the length of the RNA-DNA hybrid to be between 8 and 14 nucleotides. More recently, our group used the self-cleaving transcript assay on the elongation complex of $E$. coli RNA polymerase to determine that 12-13 nucleotides of the nascent RNA transcript are sequestered within the ternary complex as part of the RNA-DNA hybrid (Monforte et al., 1990). Presented here is the extension of this third method to the study of the T7 RNA polymerase complex.

The self-cleaving transcript assay addresses the exact point at which an RNA transcript becomes free to form structure with itself, and whether this structure is capable of forming immediately adjacent to the RNA-DNA hybrid or interacts with the hybrid to disrupt it. This simple, nonintrusive assay for structure formation uses a self-cleaving "hammerhead" RNA transcript as the complex probe. The assay accesses the elongation complex rather than an initiation complex.

The self-cleaving "hammerhead" structure is common among viroids and virusoids as well as other RNA molecules and promotes cleavage at a specific phosphodiester bond (Keese \& Symons, 1987; Prody et al., 1986; Ruffner et al., 1990). The autolytic cleavage reaction of the "hammerhead" RNA requires the formation of a specific secondary and tertiary structure, providing a powerful assay for structure formation. In order for cleavage to occur in a nascent transcript, folding of the "hammerhead" sequence must be free of constraints imposed by the ternary complex. By determining the transcript length necessary for cleavage, one can measure the length of RNA between the site of polymerization and the region of unhindered structure formation. Use of the transcript itself as an RNA structure reporter avoids artifacts potentially introduced by exogenous chemical or enzymatic probes.

The requirements for self-cleavage are "hammerhead" structure formation within the nascent transcript and the presence of magnesium ions, allowing the studies to be performed in standard transcription buffers. Cleavage is rapidly and conveniently stopped by the addition of EDTA.

The simplicity of the method allows the assay to be transported to virtually any RNA polymerase system which has a known promoter sequence and a specific initiation site. Here we present the results of the application of the self-cleaving transcript method to T7 RNA polymerase. Our results demonstrate that for T7 RNA polymerase the RNA transcript is not free of interactions in the ternary complex until it is at least 10 nucleotides away from the site of polymerization.

\section{Materials and Methods}

Materials. Purified T7 RNA polymerase was purchased from Pharmacia. Ribonucleoside triphosphates, $3^{\prime}$-deoxyribonucleoside triphosphates ( $\left.3^{\prime}-\mathrm{dNTPs}\right)$, and ribonucleases were purchased from Pharmacia. Ribonuclease inhibitor (RNasin, human placenta) was from Promega Biotec or Pharmacia. Adenosine $5^{\prime}-\left[\gamma^{-32} \mathrm{P}\right]$ triphosphate was from New England Nuclear, and T4 DNA ligase was from Amersham. Cloned T4 polynucleotide kinase was supplied by New England Biolabs. Guanylyl $\left(3^{\prime}, 5^{\prime}\right)$ guanosine $(\mathrm{GpG})$ was purchased from Sigma. Electrophoresis grade acrylamide, bis(acrylamide), ammonium persulfate, and TEMED were obtained from Bio-Rad. Ultrapure urea was from Schwarz/Mann Biotech. Diethyl pyrocarbonate treated water, silanized Eppendorf tubes, and deionized formamide were used throughout.

DNA Templates. A 127-nucleotide transcription template containing a class III T7 promoter followed by the self-cleaving transcript (Figure 1) was synthesized from DNA oligomers 45-57 nucleotides in length by standard ligation procedures (Maniatis et al., 1982). This 127 -nucleotide fragment containing the $\mathrm{T} 7$ promoter was next inserted into the polylinker 
region of puc19, which had been restricted with BamHI and $E c o R I$, and the resulting recombinant DNA was used to transform $E$. coli $\mathrm{HB} 101$. The desired plasmid was isolated by standard procedures and then digested with EcoRI to linearize the plasmid, producing a run-off transcription template.

In Vitro Transcription Reactions. All transcripts were initiated using $\left[\alpha-{ }^{32} \mathrm{P}\right] \mathrm{pGpG}$ so that the $5^{\prime}$ end of the RNA was labeled. The dinucleotide $\mathrm{GpG}$ was kinased according to the method of Monforte et al. (1990). The kinase was heat-killed for $3 \mathrm{~min}$ at $65^{\circ} \mathrm{C}$, and the buffer conditions were adjusted to those appropriate for transcription by T7 RNA polymerase by the addition of a conversion buffer. This mixture was further diluted by the addition of DNA template, RNA polymerase, and unlabeled GpG (final concentration 72 $\mu \mathrm{M})$, and this mixture was then aliquoted to the individual reaction tubes.

Transcription reactions were performed at $37^{\circ} \mathrm{C}$ for $20 \mathrm{~min}$ in 5- $\mu \mathrm{L}$ volumes in T7 transcription buffers: $40 \mathrm{mM}$ Trisacetate, $\mathrm{pH} 8.0,10 \mathrm{mM} \mathrm{NaCl}, 1 \mathrm{mM}$ spermidine, $0.01 \%$ Triton X, $10 \mathrm{mM}$ DTT, and $15 \mathrm{mM} \mathrm{MgCl}_{2}$. T7 RNA polymerase was at a concentration of $50 \mathrm{nM}$, and the DNA template was in $50 \%$ excess, being $75 \mathrm{nM}$ per lane. The concentration of NTPs ranged from $50 \mu \mathrm{M}$ to $1 \mathrm{mM}$ (actual concentrations in figure legends). The chain-terminating 3 -dNTPs were in the range of 5-100 $\mu \mathrm{M}$.

T7 RNA polymerase and DNA were added to a $\left[\alpha{ }^{32} \mathrm{P}\right]-$ pGpG mixture containing unlabeled $G p G, R N a s i n$, and the appropriate buffer. This was incubated for $8 \mathrm{~min}$ and then aliquoted into Eppendorf tubes containing various nucleoside triphosphates and chain terminator mixtures. The reaction mixtures were then incubated for $20 \mathrm{~min}$, and the transcription reaction was terminated by the addition of an equal volume of formamide containing $20 \mathrm{mM}$ EDTA and dyes. These were then analyzed by electrophoresis on denaturing $8 \mathrm{M}$ urea-12\% polyacrylamide gels (Monforte et al., 1990).

In experiments where the ternary complex was disrupted and the released transcripts self-cleave, the reaction mixture was boiled for $1.5 \mathrm{~min}$, rapidly cooled to room temperature, and incubated for $30 \mathrm{~min}$. The formamide-EDTA solution was added, and the mixture was analyzed.

\section{RESULTS}

The objective of the experiment was to obtain information on structure formation in the nascent RNA during elongation by T7 RNA polymerase. Structure formation in the RNA is influenced by steric interactions between the nascent RNA and the RNA polymerase and by the length of the RNADNA hybrid, and therefore can report on the structure of the ternary complex. The self-cleaving transcript assay allows us to easily measure the length of RNA between the site of polymerization and the region of unhindered structure formation.

These experiments require that the time scales for transcription elongation, RNA structure formation, and RNA self-cleavage be much shorter than the time required for the experiment, and disassembly of the ternary complex must be slower than all of these processes. Elongation by one base takes $4.3 \mathrm{~ms}$ for T7 RNA polymerase (Golomb \& Chamberlain, 1974). The rate of RNA structure formation is comparable, also in the millisecond range (Puglisi, 1989). The half-life of the isolated hammerhead is less than 30 s (Monforte et al., 1990). Under conditions used here, the T7 RNA polymerase ternary complex is stable for the course of an hour (Sastry \& Hearst, 1991), and evidence presented below confirms that the ternary complex is stable for the course of the experiment. Once transcription has been stalled by incorporation of chain terminators, the RNA structure has ample time to equilibrate during the course of the experiment. Therefore, a 30-min experiment provides a clear picture of the extent of cleavage at each position and the influence of the ternary complex on cleavage.

Transcriptions were performed in the presence of chainterminating nucleotides producing a sequencing ladder of stalled complexes. Only RNA molecules which had been initiated with $\left[\alpha-{ }^{32} \mathrm{P}\right] p \mathrm{ppG}$ were detected. For cleaved RNA transcripts, only the $5^{\prime}$-cleavage products are visible. Transcription reactions were quenched with a mixture of formamide and EDTA in order to simultaneously disrupt the ternary complex and prevent further "hammerhead" cleavage by denaturing the RNA and sequestering all divalent cations.

Two different templates were used, one coding for the "hammerhead" sequence and one control containing a single-base change within the "hammerhead" consensus sequence (Figure 1). The control template produces a transcript which does not cleave. The templates used were indentical to the ones used to study $E$. coli RNA polymerase except that a T7 promoter has replaced the $E$. coli Tac 18 promoter.

Figure 2 shows a typical autoradiogram of sequencing reactions performed on the "hammerhead" and noncleaving control templates. The control gives a standard sequencing pattern of stalled complexes for the full length of the DNA template (lanes 1-5). The pattern of stalled complex transcripts from the "hammerhead" (lanes 6-10) is identical to the pattern from the control template for the first $42 \mathrm{nu}$ cleotides after which the pattern changes due to transcript self-cleavage. The strong band at $\mathrm{C}_{43}$ (lanes 6-15) is the cleavage product $\left(C_{43}\right.$ indicates the transcript of length 43 , whose last base is a $C$ ). From $A_{44}$ to $U_{55}$, the pattern is similar again to the control template, indicating that transcripts from the "hammerhead" template are not efficiently cleaved in this range. Beyond $\mathrm{U}_{55}$, the band intensity is dramatically reduced and is almost negligible, indicating nearly $100 \%$ cleavage in complexes of longer transcript lengths. Certain bands between $\mathrm{G}_{43}$ and $\mathrm{C}_{56}$ have diminished intensity, most notably $\mathrm{C}_{49}$. The diminished intensity of these bands indicates a greater level for cleavage in these transcript lengths. This may be due to the particular sequence of the RNA in these ternary complexes which makes them unusually unstable, causing the complex to fall apart, releasing free RNA which then self-cleaves before the conclusion of the experiment.

The heat/cool experiment of lanes 11-15 demonstrates that the integrity of the ternary complex is essential for the prevention of self-cleavage. When the ternary complex is disrupted by heating, the RNA transcript is released, and the bands above $G_{45}$ disappear. Molecules long enough to form 3 or more base pairs of the final "hammerhead" stem selfcleave. The final "hammerhead" stem is the stem pairing the $5^{\prime}$ end of the transcript with the last section of "hammerhead" sequence to emerge from the ternary complex (Figure 1). This stem can form 6 base pairs, but formation of only the 3 innermost base pairs is required for self-cleavage in the minimum "hammerhead" structure. The heat/cool experiment demonstrates that the ternary complex is stable over the course of the experiment because released RNA molecules would rapidly self-cleave. The presence of ternary complex is essential for noncleavage of transcripts.

The cutoff position is reproducible and is obtained for a wide range of concentrations of NTPs and dNTPs. It is not altered whether a supercoiled or linearized plasmid is used. Temperature studies indicated that the same cutoff is obtained at 30 and $37^{\circ} \mathrm{C}$. At $23^{\circ} \mathrm{C}$, several intermediates of lengths 


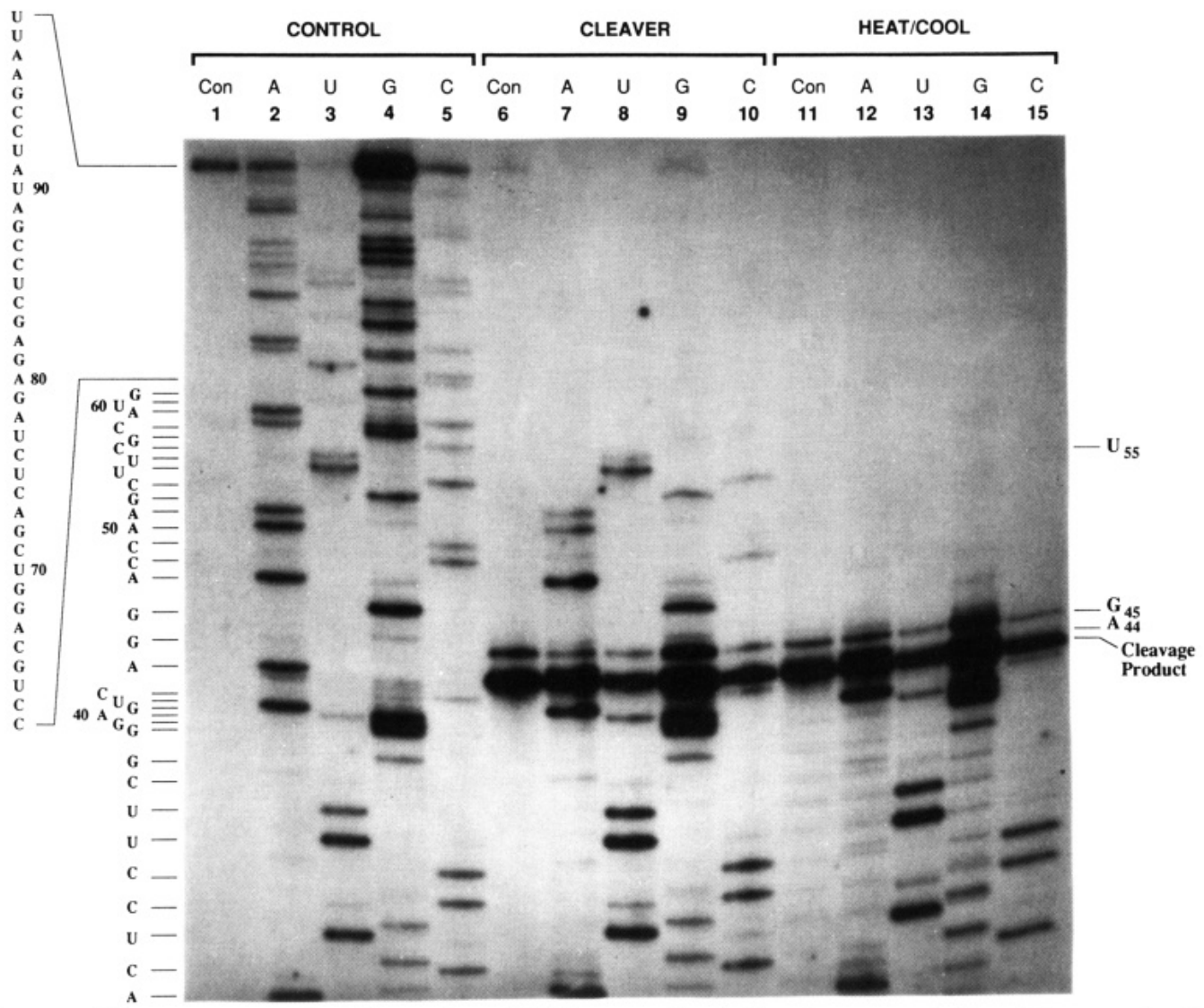

FIGURE 2: Self-cleavage of the nascent transcript. Comparison of RNA sequencing ladders from transcription of the control and "hammerhead" templates shows self-cleavage of the transcript. Lanes 1-5 show transcription on the control template in the presence of four different chain terminators. Lanes 6-10 are equivalent to lanes 1-5 except the "hammerhead" template coding for the self-cleaving transcript is used. Lanes 11-15 are identical to lanes 6-10 except that it shows transcript self-cleavage after disruption of the ternary complex. Transcription was performed at $37^{\circ} \mathrm{C}$ for $30 \mathrm{~min}$. The triphosphate concentrations used in each lane are as follows: Control and "hammerhead" experiments (lanes 1-15). Control (Con) lanes: ATP, UTP, and CTP, $400 \mu \mathrm{M}$; GTP, $50 \mu \mathrm{M}$. U lane: 3'-dATP, $100 \mu \mathrm{M}$; ATP, $400 \mu \mathrm{M}$; GTP, $50 \mu \mathrm{M}$; CTP and UTP, $1 \mathrm{mM}$. U lanes: 3'dUTP, $50 \mu \mathrm{M}$; UTP, $400 \mu \mathrm{M}$; GTP, $50 \mu \mathrm{M}$; CTP and ATP, $1 \mathrm{mM}$. G lanes: 3'-dGTP, $5 \mu \mathrm{M}$; GTP, $100 \mu \mathrm{M}$; UTP, ATP, and CTP, $1 \mathrm{mM}$. C lanes: $3^{\prime}$-dCTP, $50 \mu \mathrm{M} ; \mathrm{CTP}, 400 \mu \mathrm{M}$; GTP, $50 \mu \mathrm{M}$; UTP and ATP $1 \mathrm{mM}$. In lanes 6-15, transcription was performed on "hammerhead" template at $37^{\circ} \mathrm{C}$ for $20 \mathrm{~min}$ at which time the reaction volumes were split. For lanes $6-10$, the reactions were immediately quenched with EDTA/formamide. For lanes $11-15$, the reactions were heated to $90^{\circ} \mathrm{C}$ for 2 min to disrupt the complex and release the transcripts, quick-cooled to $22{ }^{\circ} \mathrm{C}$, incubated for $30 \mathrm{~min}$, and then quenched. This procedure allows free transcripts of sufficient length to self-cleave.

between 56 and 99 nucleotides are observable (data not shown). This could be attributed to a slower rate of cleavage, due to the stabilization of alternate conformations, at this temperature. Time course studies of the cleavage reaction show that the ternary complex is stable for more than $20 \mathrm{~min}$ and that the same cutoff is obtained at different time points (data not shown).

Within the T7 ternary complex, 56 nucleotides of the RNA transcript must be transcribed in order for self-cleavage to occur. Of these 56 nucleotides, 46 are required to be free of the ternary complex, allowing the minimum "hammerhead" structure to form and afford cleavage (Figure 3). This result demonstrates that the formation of minimum "hammerhead" structure and self-cleavage of transcripts are inhibited for RNA molecules within the ternary complex up to $55 \mathrm{nu}$ cleotides in length. Self-cleavage of the transcripts occurs only when 10 nucleotides have been synthesized beyond $G_{46}$. A maximum of 10 nucleotides of the nascent transcript are unavailable for RNA-RNA base pair formation, beyond which the transcript is free to form structure.

\section{Discussion}

Our data clearly demonstrate that in the RNA.DNA.T7 RNA polymerase ternary complex the formation of intramolecular structure in the nascent RNA transcript is inhibited for the first 10 nucleotides upstream of the site of polymeri-

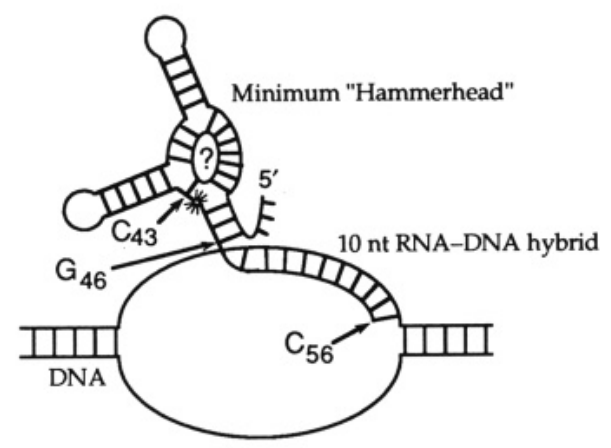

FIGURE 3: Model of the ternary complex at the point of self-cleavage. The cleaving, 56-nucleotide-long, nascent RNA transcript, the DNA of the transcription bubble, and the RNA-DNA hybrid are shown. The $3^{\prime}$ end of the transcript is involved in the proposed 10-nucleotide RNA-DNA hybrid. Ten nucleotides beyond the site of polymerization, the RNA becomes free of the RNA-DNA hybrid and the ternary complex and forms the minimum "hammerhead" structure with a 3 base pair final stem. The cleavage point is indicated between nucleotides 43 and 44 . The question mark represents the tertiary structure of the "hammerhead".

zation whereas beyond 10 nucleotides RNA structure can form freely. The nascent RNA is restrained by interactions with components of the ternary complex, either due to formation of an RNA-DNA hybrid or due to steric interactions with the RNA polymerase. The assay for RNA structure formation 
is the self-cleavage of a "hammerhead" transcript sequence as its structure forms upon emergence from the ternary complex.

The data suggest that the maximum possible length of the RNA-DNA hybrid for T7 RNA polymerase is 10 nucleotides. This is distinctly different from the 12-nucleotide RNA-DNA hybrid length currently accepted in the literature for $E$. coli RNA polymerase which was also confirmed using this same self-cleaving transcript assay (Monforte et al., 1990). The data imply that a 12-nucleotide RNA-DNA hybrid is not a fundamental feature of the transcription apparatus and may vary depending on the size and complexity of the RNA polymerase. They also introduce a new minimum to the possible length of the RNA-DNA hybrid and suggest that an elongating ternary complex can be stabilized by a shorter, 10-nucleotide RNADNA hybrid. The data fit in with the picture of T7 RNA polymerase being a "minimum apparatus" for transcription.

Extension of these studies to the more complex eukaryotic RNA polymerases would prove informative. Recent RNase digest experiments have indicated that 18-22 nucleotides are protected from RNase digestion by Simian RNA Pol II (Choder \& Aloni, 1988). This result suggests either that the length of RNA protected due to interactions in the ternary complex is much larger than in E. coli or T7 RNA polymerase or that large exogenous probes like RNase are sterically excluded from the site of transcript emergence. While such an experiment can provide an outer limit to the length of protected RNA, it cannot clearly identify the point at which the RNA is able to interact with itself and where polymerase-specific, transcript binding accessory factors will have access to the nascent transcript.

Footprinting studies by Ikeda and Richardson (1986) have shown that the conformation of T7 RNA polymerase is different after 15 nucleotides of synthesis than it is after the first 6 nucleotides. These data imply that a switch between initiation and elongation modes must occur somewhere in this range. Studies of abortive initiation by T7 RNA polymerase (Martin et al., 1988) show that the maximum length of abortive transcription is eight nucleotides, suggesting that an abrupt change in the stability of the ternary complex occurs upon incorporation of ninth nucleotide. It is likely that the transition to a stable elongation complex occurs at 9 or at most 10 nucleotides into transcription, since beyond 10 nucleotides the RNA exits from the transcription complex.

Studies on isolated stalled complexes of $E$. coli RNA polymerase have shown that when the RNA transcript length is increased from 8 to 10 nucleotides the RNA polymerase exhibits a change in conformation and loss of the $\sigma$ factor; this corresponds to a shift from the initiation complex to the more stable elongation mode (Krummel \& Chamberlin, 1989). The stabilization of the ternary complex occurs when the transcript length is about 9-10 nucleotides, even though the length of the RNA-DNA hybrid in the elongation mode is 12 nucleotides. This suggests that an RNA-DNA hybrid length of 10 nucleotides is sufficiently long to stabilize the ternary elongation complex of $E$. coli RNA polymerase.

The length of 10 nucleotides is significant in the stabilization of the ternary elongation complexes of both T7 RNA polymerase and $E$. coli RNA polymerase. This length represents the minimum and sufficient length of RNA needed for stabilization of the RNA polymerase ternary complexes in the stable elongation mode and may represent the transition point from initiation to elongation for all RNA polymerases. For polymerases which share homology with T7 RNA polymerase, such as T3, SP6, and yeast mitochondrial RNA polymerases, it is very likely that they have a similar 10-nucleotide RNADNA hybrid and initiation to elongation transition point. Extension of the length of the RNA-DNA hybrid by polymerases such as $E$. coli RNA polymerase promotes increased complex stability and allows for more refined control over different aspects of transcription regulation, especially termination. Such fine-tuning is not required by simple viral systems such as $\mathrm{T} 7$.

\section{ACKNOWLEDGMENTS}

We thank members of the Hearst lab for helpful advice and discussion, especially Srinivas Sastry for the information about T7 RNA polymerase ternary complex stability. David Koh synthesized all DNA oligonucleotides. David Cook and Dzwokai Ma assisted in plasmid preparation.

Registry No. T7 RNA polymerase, 9014-24-8; hammerhead sequence, 136658-57-6.

\section{REFERENCES}

Chamberlin, M., \& Ryan, T. (1982) Enzymes (3rd Ed.) 15, 87-108.

Choder, M., \& Aloni, Y. (1988) J. Biol. Chem. 263, 12994-13002.

Coleman, J. E. (1974) Biochem. Biophys. Res. Commun. 60, 641-648.

Fedor, M. J., \& Uhlenbeck, O. C. (1990) Proc. Natl. Acad. Sci. U.S.A. 87, 1668-1672.

Gamper, H. B., \& Hearst, J. E. (1982) Cell 29, 81-90.

Golomb, M., \& Chamberlin, M. (1974) Biochemistry 23, 2858-2863.

Hanna, M. M., \& Meares, C. F. (1983) Proc. Natl. Acad. Sci. U.S.A. $80,4238-4242$.

Ikeda, R. A., \& Richardson, C. C. (1986) Proc. Natl. Acad. Sci. U.S.A. 83, 3614-3618.

Keese, P., \& Symons, R. H. (1987) in Viroids and Viroid-Like Pathogens (Semancik, J. S., Ed.) pp 1-47, CRC Press, Boca Raton, FL.

Krummel, B., \& Chamberlin, M. J. (1989) Biochemistry 28, 7829-7842.

Kumar, S. A., \& Krakow, J. J. (1975) J. Biol. Chem. 250 , 2878-2884.

Maniatis, T., Fritsch, E. F., \& Sambrook, J. (1982) Molecular Cloning: A Laboratory Manual, Cold Spring Harbor Laboratory, Cold Spring Harbor, NY.

Martin, C. T., Muller, D. K., \& Coleman, J. E. (1988) Biochemistry 27, 3966-3974.

Masters, S. B., Strohl, T. L., \& Clayton, A. D. (1987) Cell 51, 89-99.

Monforte, A. J., Kahn, D. J., \& Hearst, E. J. (1990) Biochemistry 29, 7882-7890.

Prody, G. A., Bakos, J. T., Buzayan, J. M., Schneider, I. R., \& Bruening, G. (1986) Science 231, 1577-1580.

Puglisi, J. D. (1989) RNA Folding: Structure and Conformational Equilibria of RNA Pseudoknots, Dissertation, University of California, Berkeley.

Ruffner, D. E., Stormo, G. D., \& Uhlenbeck, O. C. (1990) Biochemistry 29, 10695-10702.

Sastry, S. S., \& Hearst, J. E. (1991) J. Mol. Biol. (in press). Shi, Y.-B., Gamper, H., \& Hearst, J. (1988) J. Biol. Chem. 266, 527-534.

von Hippel, P. H., Bear, D. G., Morgan, W. D., \& McSwiggen, J. A. (1984) Annu. Rev. Biochem. 53, 389-446.

Yager, T. D., \& von Hippel, P. H. (1991) Biochemistry 30, $1097-1118$. 